\title{
Case report \\ Ewing's sarcoma of the distal part of ulna treated by wide excision and reconstruction by non-vascularized autogenous fibular graft: A case report.
}

\author{
Arefin KN', Mahmud SA ${ }^{2}$, Khatun $M^{3}$, Hoque $\mathrm{MJ}^{4}$ Zaman $\mathrm{ME}^{5}$,Rahman $\mathrm{A}^{6}$
}

\begin{abstract}
Abstruct:
Ewing's sarcoma of the bone is the second most frequently occurring malignant bone tumor in children and adolescents. Ewing's sarcoma in the distal ulna is extremely rare. Thus, the surgical options for reconstruction of the ulna are limited and technically challenging. It can be treated with different methods of reconstruction. Wide local excision following neoadjuvant chemotherapy followed by reconstructive procedure is the usual method of limb salvage surgery. Patient needs post operative adjuvant chemotherapy and local radiotherapy to reduce the chance of local recurrence and systemic metastasis. We present a case of Ewing sarcoma of distal end of ulna in a 9 years girls treated with limb salvage surgery as neoadjuvant chemotherapy followed by wide excision and reconstruction with non vascularised fibular graft and post operative chemotherapy and local radiotherapy. Graft was uniting well and without any local recurrence or systemic metastasis at subsequent follow up to 6 months. There was no neurovascular and functional deficit occurred.
\end{abstract}

\section{Key words: Ewing's sarcoma, stabilization, resections, non-vascularized fibular graft, ulna, Bone tumor.}

\section{Introduction:}

Ewing's sarcoma of the bone is the second most frequently occurring malignant bone tumor in children and adolescents. It is a member of the Ewing's sarcoma family of tumors, which also includes primitive neuroectodermal tumors, Ewing's soft tissue sarcomas and Askin's tumors. The Ewing's sarcoma family of tumors is high-grade aggressive lesions that most commonly originate in the bone and are associated with large soft tissue masses and frequent metastases. The majority of Ewing's sarcomas of the bone are located in the lower extremities and pelvic girdle, but occasionally arise in the ulna ${ }^{1}$. Previous treatments for Ewing's sarcomas, such as surgery (alone), radiotherapy or monochemotherapy, have failed to achieve ideal results. The majority of patients succumbed within two years and the fiveyear survival rate was $<20 \%{ }^{2}$. However, with progress in chemotherapy, the prognosis for patients with Ewing's sarcoma has improved considerably during the past three decades $^{3}$. Currently, chemotherapy and surgery are the standard treatment for Ewing's sarcomas ${ }^{4}$. The ulna is an uncommon site for these malignant and aggressive tumors. Thus, the surgical options for reconstruction are limited and technically challenging ${ }^{4-8}$. The current study presents the case of a successful wide excision and reconstruction using a non-vascularized, autogenous fibular graft in a 9-year-old girl with Ewing's sarcoma of the ulna. Written informed consent was taken from the patient's gurdian.

\section{Case report:}

A 9-year-old, healthy appearing, right-hand dominant girl, presented with an one and half -month history of painless, increasing swelling along the ulnar aspect of his left distal forearm. On examination the swelling was diffusely tender and its consistency was firm. The overlying skin colour was normal, free from under lying structure. Temperature over the swelling was raised. Tenderness was preset. Nerurovascular status was intact. Range of movement of left wrist, elbow and shoulder within normal and painless. The grasping power was equal in both hands. There was no evidence of lymphadenopathy and no known systemic disease. Systemic examinations did not reveal any abnormality.

1. Khondokar Nurul Arefin, Associate Professor, Department of Orthopaedic surgery, BSMMU.

2. Sayeed al mahmud, MO, Narsingdi Sadar Hospital.

3. Mahbuba Khatun, OSD surgery, BSMMU.

4. Md. Johurul Hoque, Associate Professor, Department of Orthopaedic surgery, ZH Sikder Womens Medical College, Dhaka.

5. Muhammad Emam-Uz-Zaman, D-Ortho, Registrar, Department of Orthopaedic surgery, ZH Sikder Womens Medical College, Dhaka.

6. Azizur Rahman, MO, Faridpur Medical college Hospital.

\section{Address of Correspondence:}

Muhammad Emam-Uz-Zaman, D-Ortho, Registrar of Department of Orthopaedic surgery, ZH Sikder Womens Medical College, Dhaka. Email: zaman.mmc45@gmail.com 


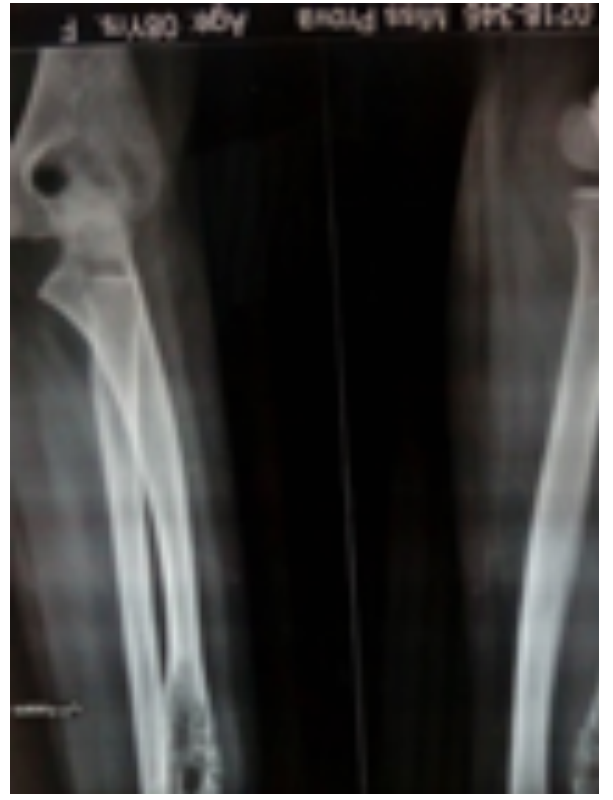

Figure 1: $x$-ray left forearm lateral and AP view showing fusiform shaped mixed lesion with cortical breech in lower end of ulna.

Haematological and biochemical investigations were normal. A plain radiograph demonstrated a mixed osteolytic lesion with cortical destruction involving the middle and distal ulna (Fig. 1). Magnetic resonance imaging demonstrated an intramedullary mass lesion $(6.7 \times 2.6 \times 2.4 \mathrm{~cm})$ that involved the lower diaphyseal and metaphyseal region of the ulna, with cortical breech and large soft tissue component with irregular interrupted periosteal reaction (Fig-2)

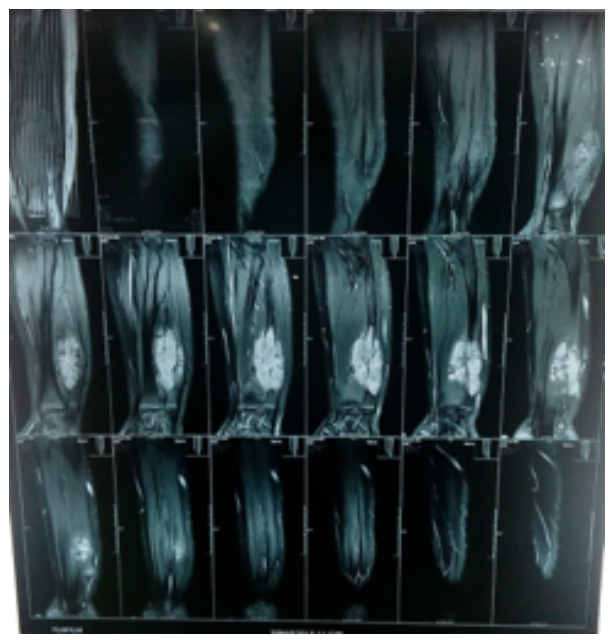

Figure 2: MRI showing an intramedullary mass lesion ( $6.7 \times 2.6 \times 2.4 \mathrm{cms}$ ) that involved the lower diaphyseal and metaphyseal region of the ulna.

The surrounding neurovascular structure was not involved. On $27^{\text {th }}$ april FNAC was done and reveals malignant tumor cells are round to oval even spindle shaped and have attempted at rosette formation in other areas. Then the patient underwent a core biopsy, and histopathology examination demonstrated malignant tumor made of small round cells arranged in sheets separated by fibrous septa with finely dispersed nuclear chromatin and inconspicuous nucleoli. Subsequently, immunohistochemistry revealed that the cell membrane was strongly positive for cluster of differentiation ${ }^{99}$ and negative for Desmin. Thus, the analysis of the tumor biopsy supported diagnosis of Ewing's sarcoma. Bone scan revealed uptake only in the left ulna and chest x-ray showed no evidence of metastatic disease. Therefore, the patient was classified as having stage IIB according to the Enneking surgical staging system. Then the patient received 6 cycle chemo twice weekly interval including Ifosfamide, Etoposide, Vincristin, Cycloposphamide and doxorubicin. As the tumor involved distal half of the ulna, patient underwent a wide excision of the ulna except proximal $3^{\text {rd }}$ of the ulna (fig-3).

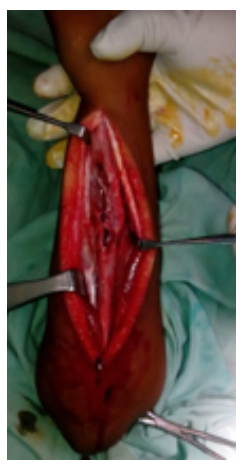

Figure 3: per operative picture showing tumor mass in distal ulna.

Reconstruction of the bony defect was performed by using a non-vascularized, autologous fibular graft harvested from the ipsilateral leg (fig-4).

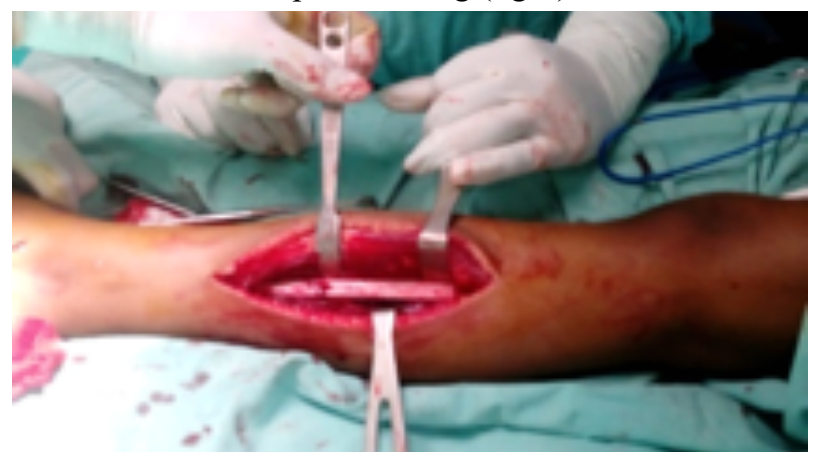

Figure 4: Graft harvesting from fibula

Then the fibular graft was fixed with ulna by a Kirschner wire (fig-6). Post-operatively chemotherapy was given as 
schedule. Post operative rehabilitation included 4 weeks of full immobilization by above elbow POP cast at $90^{\circ}$ flexion. Then followed by passive and active assisted flexion and extension of the left elbow was done.

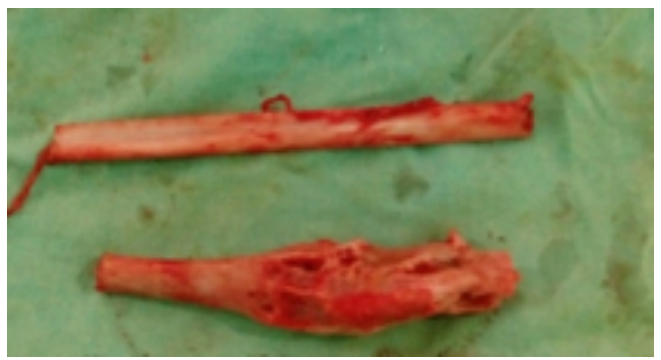

Figure 5: Excised Fibular graft (a) and tumor mass from ulna (b)

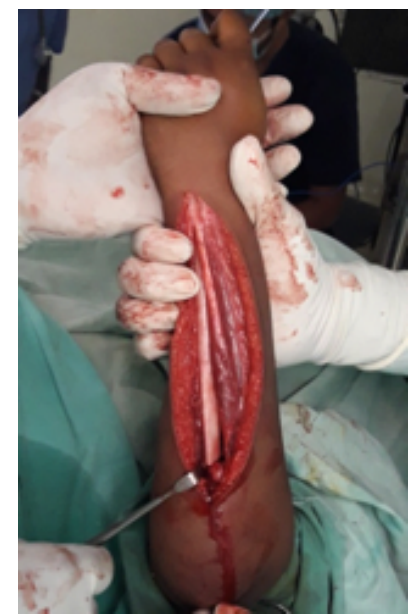

Figure 6: Figure 6: Reconstruction of ulna by nonvascularized fibular graft.

Graft was uniting well (fig 7), without any local recurrence or systemic metastasis in the subsequent follow up. There was no neurovascular and functional deficit. No morbidity in the donor site was observed and the functional capability of the leg was good.
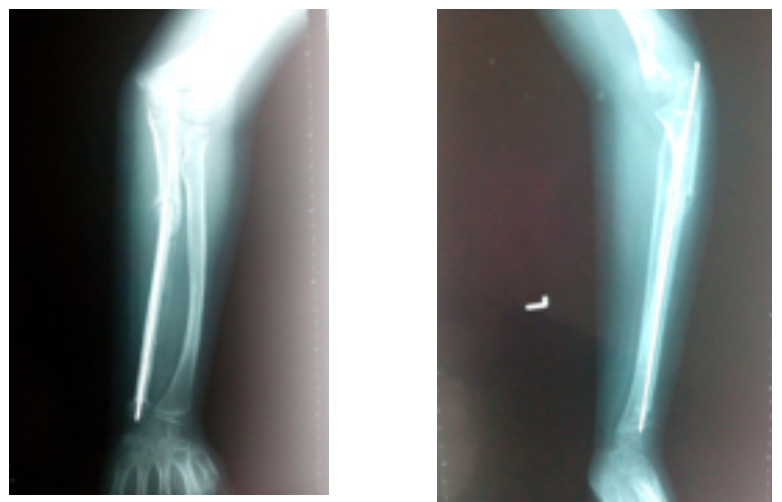

Figure 7: x-ray showing uniting fibular graft in ulna with $K$ wire in situ.

5 months later, patient was found well, active and able to perform to her daily activities freely.

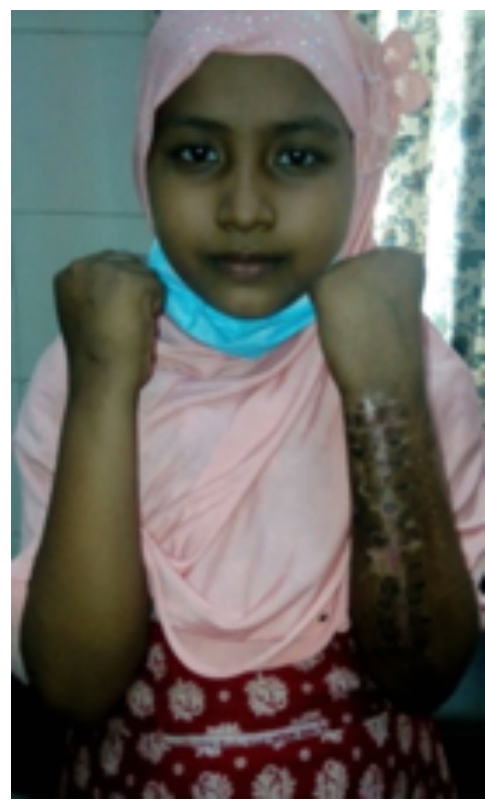

Figure 8: After 5 month, ROM of elbow and wrist normal.

\section{Discussion}

Ewing sarcoma involving long bone is common and most common primary malignancy in less than 10 years

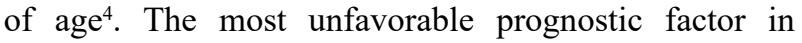
Ewing's sarcoma is the presence of distant metastasis at the time of diagnosis ${ }^{7}$. Even with aggressive treatment, patients with metastases have only an approximately $20 \%$ chance of long-term survival ${ }^{8}$. Involvement of the ulnar diaphysis with this tumor posses challenge to surgeon for limb salvage surgery. If expertise and resources are limited excision through normal plane after neo-adjuvant chemotherapy and reconstruction with nonvascularised diaphysis of the fibula is viable option. Other options for reconstructions are vascularized fibular graft which demands much more operative time, microsurgery expertise and vascular instrument sets.

Chemotherapy most commonly used to treat Ewing sarcoma regardless of their identification at initial staging includes doxorubicin (DXR), cyclophsophamide (CPA), vincristine (VCR), actinomycin-D (ACT), ifosfamide (IFM), and etoposide (VP16) ${ }^{3}$. As Ewing's sarcomas are sensitive to both chemotherapy and irradiation, even questionable candidates for limb salvage may be eligible after neoadjuvant chemotherapy with or without irradiation. If the surgical margins are found to be inadequate after surgery, postoperative radiotherapy is added. When surgical margins are certain to be inadequate at preoperative imaging, amputation is the only surgical option available. 


\section{Conclusion:}

In this case, early diagnosis of Ewing, sarcoma of ulnar diaphysis and metaphysis which was initially treated with neoadjuvant chemotherapy and followed by limb salvage surgery as wide excision and reconstruction with non vascularised fibular graft and postoperative chemotherapy showed satisfactory results in well being and functional state of limbs.

\section{References}

1. Moore DD and Haydon RC: Ewing's sarcoma of bone. Cancer Treat Res 162: 93-115, 2014.

2. Widhe B and Widhe T: Initial symptoms and clinical features in osteosarcoma and Ewing sarcoma. $2000 \mathrm{~J}$ Bone Joint Surg Am 82: 667674, Cotterill SJ, Ahrens S, Paulussen M, Jürgens HF, Voûte PA, Gadner H and Craft AW: Prognostic factors in Ewing's tumor of bone: Analysis of 975 patients from the European intergroup cooperative Ewing's Sarcoma study group. $2000 \mathrm{~J}$ Clin Oncol 18: 31083114.

3. Wunder JS, Paulian G, Huvos AG, Heller G, Meyers PA and Healey JH: The histological response to chemotherapy as a predictor of the oncological outcome of operative treatment of Ewing sarcoma. 1998 J Bone Joint Surg Am 80: 10201033,

4. Enneking WF: A system of staging musculoskeletal neoplasms. 1986 Clin Orthop Relat Res 204: 924.

5. Sułko J: Elbow reconstruction following an extensive resection of the proximal part of the ulna in a patient with Ewing Sarcoma. A case report. 2013 JBJS Case Connect 3: e111.

6. S Pandey and S Pokharel: ewing sarcoma of ulna treated with resection and reconstruction with fibula: a case report. Journal of Chitwan Medical College; 2012, 1(2); 63-64 .

7. KAYIAS et al :Resection of the distal ulna for tumours and stabilisation of the stump. A case report and literature review. Acta Orthop. Belg., 2006, 72, 484-491 